\title{
Association between serum chemokine CC-motif ligand 17 and prognosis of patients at risk for coronary heart disease
}

\section{Siqin Feng}

Peking Union Medical College Hospital

\section{Lei Wang}

Peking Union Medical College Hospital

\section{Yifei Wang}

Tsinghua University School of Medicine

\section{Yiyang Wang}

Tsinghua University School of Medicine

\section{Yuxiong Chen}

Peking Union Medical College Hospital

\section{Ran Tian}

Peking Union Medical College Hospital

Hui Wang

Peking Union Medical College Hospital

Honghong Liu

Peking Union Medical College Hospital

Shuyang Zhang ( $\nabla$ shuyangzhang103@nrdrs.org)

Peking Union Medical College Hospital https://orcid.org/0000-0002-1532-0029

\section{Original investigation}

Keywords: Atherosclerosis, Coronary heart disease, Chemokine CC-motif ligand 17, Inflammation

Posted Date: January 29th, 2021

DOI: https://doi.org/10.21203/rs.3.rs-154494/v1

License: (c) (i) This work is licensed under a Creative Commons Attribution 4.0 International License. Read Full License 


\section{Abstract}

Background It has been reported chemokine CC-motif ligand 17 (CCL17), an inflammatory mediator modulating $T$ cells function to affect formation and stableness of atherosclerotic plaque and is associated with the severity of coronary heart disease (CHD). This research aims to further explore the relationship between serum CCL17 and the prognosis of patients at risk for CHD. Method This study retrospectively enrolled 495 patients receiving coronary angiogram at Peking Union Medical College Hospital from January 2012 to December 2013. Serum CCL17 was measured on admission and annual routine telephone follow-up was carried out till December 2020. The prognosis of patients was evaluated by the incidence of major adverse cardiovascular events (MACE). Cox survival analysis have been applied for univariate and multivariate models setting., Machine learning and data imbalance methods were also added to improve the model performance. Results Compared with patients with low serum CCL17 level, patients with medium and high serum CCL17 demonstrated elevated risk to develop MACE, with the hazard ratio (HR) being 4.53 [95\% confidence interval $(\mathrm{Cl})$ 2.08-9.88] and 6.38 (95\% Cl 3.07-13.28) respectively. CCL17 served as an independent risk factor to predict the incidence of MACE and the addition of CCL17 to the baseline risk model significantly increase the predictive ability for MACE (Area under curve: baseline risk model $0.803 \pm 0.021$ vs baseline risk model+CCL17 $0.836 \pm 0.021, p$ for comparison<0.001). Conclusions Elevated CCL17 functions as an independent predictor for MACE in patients at risk for $\mathrm{CHD}$ and the potential mechanisms deserved more exploration in the future.

\section{Background}

Cardiovascular disease (CVD) is a group of diseases involving heart or blood vessels, including coronary heart disease (CHD), cerebrovascular disease, vein thrombosis, pulmonary embolism, etc. CVD is the leading cause of death, and around 17.8 million global death was caused by CVD in 2017 with an ageadjusted death rate of 233.1 per 100,000 people[1]. It was estimated that CVD would continue to be the leading cause of death and account for $>22.2$ million deaths by $2030[1,2]$. Among all death induced by CVDs, CHD-related death accounts for $42.6 \%$ and is a major threat to life[1]. Therefore, looking for risk factors of $\mathrm{CHD}$ is always an important task to improve the life quality of the global population.

Multiple risk factors have been reported to be associated with the pathogenesis of CHD, such as age, smoke, hypertension, hyperlipidemia, hypercoagulability, with complex mechanisms involved. Atherosclerosis is the principal cause of $\mathrm{CHD}$ [3]. The etiology of atherosclerosis includes lipid deposition on intima of coronary vessels, dysfunction of endothelial cells, infiltration of smooth muscle cells, and inflammatory response[4-7]. Chronic inflammation plays a vital role in the pathogenesis of atherosclerosis, which is substantiated by the presence of various kinds of immune cells and inflammatory cytokine in human atheroma[8, 9]. Inflammatory mediators, including chemokines, interferon-y, interleukins, and so on, were reported to modulate immune cell function, involve in lesion formation, and affect plague stableness[6, 8]. 
Chemokine CC-motif ligand 17 (CCL17), also known as thymus activation and regulated chemokine (TARC), is involved in inflammatory responses of different disease models. It is secreted by mature dendritic cells (DC)[10] and is capable of promoting Th2 skewed T cell response through binding to CCchemokine receptor 4 (CCR4)[11-13]. CCL17 was also found to be involved in multiple diseases, including atherosclerotic lesions, Kawasaki disease, multiple sclerosis, atopic dermatitis, etc $[12,14-16]$.

It has been verified that CCL17 was able to promote atherogenesis via restraining regulatory $T$ cells (Tregs) maintenance and survival in lymphatic tissues, and the knocking out of CCL17 attenuated the atherosclerotic lesions in mice[11, 12,17]. The correlation was also supported by epidemiological evidence. In patients who underwent coronary angiography, serum CCL17 concentration in CHD patients was found significantly higher than CHR patients $(265.95 \mathrm{pg} / \mathrm{mL}$ vs $218.35 \mathrm{pg} / \mathrm{mL}, \mathrm{p}<0.001)$, and the serum CCL17 concentration exhibited a linear trend with the severity of $\operatorname{CHD}[18,19]$. However, the prognostic significance of baseline CCL17 in patients at risk for CHD has not been fully studied. In this study, we aimed to explore the association between CCL17 and the prognosis of patients undergoing coronary angiography and evaluate its potency working as a predictive risk factor.

\section{Methods}

\section{Study population}

The study is a single-center, observational study focusing on 1022 inpatients receiving coronary angiography in Peking Union Medical College Hospital from January 2012 to December 2013. The patients were informed and signed informed consent about participating in a long-term follow-up research program. Patients who rejected participation or failed to offer stable contact information were excluded. Other exclusion criteria include: 1) Autoimmune disease; 2) Malignancy; 3) Current infection; 4) Asthma; 5) Dermatitis; 6) Vasculitis; 7) Abnormal liver function with alanine aminotransferase (ALT) $>3$ times of upper limit; 8) Severe renal failure with estimated glomerular filtration rate (eGFR) $<30 \mathrm{~mL} /\left(\mathrm{min}^{*} 1.73 \mathrm{~m}^{2}\right)$.

Finally, a total of 495 patients who met the enrollment principles were included in the present analyses (Figure 1).

\section{Clinical data collection and definition}

Patients' demographic information, medication, medical history, physical and laboratory results, were extracted from the discharged medical records in the electronic medical information recording system of Peking Union Medical College Hospital. Body mass index (BMI) was calculated via equilibrium: $\mathrm{BMI}=$ weight $(\mathrm{kg}) /[\text { height }(\mathrm{m})]^{2}$. Medical history, including smoking, drinking, hypertension, dyslipidemia, and diabetes, was extracted and recorded as long as available in patients' electronic medical records. The diagnostic criteria of these diseases were demonstrated in the supplementary method section of supplementary material. 
All the patients were divided into 5 groups according to their diagnosis: 1) Cardiovascular high-risk patients (CHR): patients with $<50 \%$ stenosis in the main coronary arteries did not meet the diagnostic criteria of CHD, but did in a high risk of developing CHD. Patients with $>50 \%$ stenosis were diagnosed with CHD, which could be further classified to diagnosis 2), 3), 4) and 5). 2) Stable angina pectoris (SAP): patients with classical symptoms of angina yet did not meet the diagnostic criteria of unstable angina (UA). 3) UA. The angina symptoms of patients met the following criteria: prolonged anginal pain $(>20$ $\mathrm{min}$ ) at rest, or newly onset angina, or destabilization of previous existing angina, or post-myocardial infarction angina. 4) Non-ST segment elevation myocardial infarction (NSTEMI). 5) ST-segment elevation myocardial infarction (STEMI). Myocardial infarction was defined as rising and/or fall of cardiac troponin above $99^{\text {th }}$ percentile upper reference limit for at least one time, with at least one of the following expressions: 1) Myocardial ischemia syndrome; 2) New electrocardiography (ECG) changes indicating ischemia; 3) Pathological Q waves; 4) Imaging evidence showing the loss of new viable myocardium or newly developed segmental wall motion abnormality; 5) Identification of intracoronary thrombus via angiography or autopsy[20]. Myocardial infarction patients were further diagnosed as NSTEMI or STEMI based on the results of ECG.

Coronary angiography was performed by experienced interventional cardiologists in Peking Union Medical College Hospital. Coronary angiogram data were recorded and analyzed by two experienced cardiologists with more than ten years of experience who were blind to the study. Gensini score was used to evaluate the severity of atherosclerosis and the differential diagnosis of CHR and CHD patients.

\section{Serum CCL17 quantification}

The blood sample was collected from the radial or femoral artery after sheath insertion before coronary angiography for serum CCL17 testing. The serum was isolated by centrifugation $₫ 3000 \mathrm{rpm}, 10 \mathrm{~min} \rrbracket$ and was frozen at $-80^{\circ} \mathrm{C}$ immediately until CCL 17 measurement. Serum CCL17 level was tested via the Quantikine ELISA kit for human CCL17 (R\&D Systems, Minneapolis, MN, USA) according to the manufacture's instruction. The result was read through Labsystems Multiskan MS spectrophotometer (Thermo Labsystems Oy, Helsinki, Finland).

\section{Follow-up and endpoint event}

Upon being discharged, patients that agreed to participate in the long-term research program would receive routine telephone follow-up by trained professionals blinded to patients' information annually. The follow-up was carried out till December 2020. Attention was paid to the incidence of major cardiovascular events (MACE). The information was further verified by corresponding medical records if necessary. Patients who failed to respond for 6 times consecutively, or changed the contact information, were defined as loss to follow-up.

The definition of MACE includes: 1) All-cause death; 2) Myocardial infarction; 3) Angina; 4) Heart Failure; 5) Ischemic cerebrovascular event; 6) Hemorrhagic cerebrovascular event 7) In-stent stenosis, which is defined as $>50 \%$ stenosis in-stent during follow-up; 8) Re-percutaneous transluminal coronary 
intervention (PCI); 9) Re-coronary artery bypass grafting (CABG). For a single patient with multiple MACE, only the most severe MACE was recorded, and the order is: Death $>\mathrm{Re}-\mathrm{PCl}=\mathrm{Re}-\mathrm{CABG}>\mathrm{In}$-stent stenosis $>$ Myocardial infarction $>$ Heart failure $>$ Angina $>$ Ischemic/Hemorrhagic cerebrovascular event.

\section{Statistical analysis}

As CCL17 was a continuous variable, we applied receiver-operating characteristic (ROC) curve analysis and the Youden Index, which is defined as sensitivity + specificity -1 , to divide the CCL17 into three levels by the optimal cutoff values of the curves. For statistic description of patients' baseline characteristics in each level, normally distributed continuous variables were expressed by mean \pm standard deviation, while skewed distributed variables were expressed by median (interquartile ranges, IQR). Categorical variables were expressed by number and percentage.

ANOVA, LSD, Chi-square, Wilcoxon rank tests, and Kruskal-Wallis $\mathrm{H}$ tests were applied for univariate statistical inference. The Kaplan-Meier survival analyses were performed to evaluate the incidence rate of MACE in groups of different CCL17 levels and discrepancies between groups were evaluated by the logrank test.

The predictive value of variables for MACE was evaluated through the univariate survival tests (Log-rank test and Cox proportional hazards analyses). In multivariate Cox proportional hazards regression models, three models were established to evaluate the predictive value of CCL17 for MACE, among which the confounders were selected based on statistical significance in univariate analysis and clinical importance. Additionally, to avoid multicollinearity in each model, not all variables with statistical significance were adjusted in all models while listed the details of confounders as follows. Model 1 adjusted for clinical demographics known as traditional risk factors for CHD: Age + Sex + BMI. Based on model 1, model 2 adjusted for additional disease risk factors: Age + Sex + BMI + Diabetes + Hypertension + Number of lesion vessels. Based on model 2, model 3 adjusted for laboratory results related to MACE: Age + Sex + BMl + Diabetes + Hypertension + Number of lesion vessels + PLT (platelet) + hsCRP (high sensitivity $C$ reactive protein) + eGFR (estimated glomerular filtration rate)+ ALT (alanine aminotransferase).

Besides, the model performance was assessed through C-index and Area under ROC curves (AUC). Data imbalance problem has existed in this cohort as the number of patients with MACE $(N=116)$ was more than three times smaller than that of the patients without MACE ( $N=379)$. To improve the model performance, we applied the Synthetic Minority Oversampling Technique (SMOTE) for data imbalance problems and machine learning methods for further model performance extension, including Coxboost and Random Forest (RF). According to the imbalanced data, the class with more samples were identified as the majority, and vice versa. The SMOTE is an oversampling method, which synthetic samples of the minority class by oversampling each data-point by considering linear combinations of existing minority class neighbors more samples[21]. For machine learning methods we applied, the Coxboost has added the Adaboost ensemble learning to Cox survival methods by likelihood[22]. The other survival analysis method is RF which combined tree classifiers through the Bagging ensemble learning method[23]. During 
the model improvement process, the dataset was divided into 10 parts randomly. Seven of them were combined for model training, while the rest three were left for model testing. Recycled the model setting process 1000 times, tested the model assessment indexes through a t-test to find out whether CCL17 could improve the machine learning model performance. The significance was defined as a p-value below 0.05 .

The data analysis was conducted in SPSS 25.0 (IBM corporation, Armonk, NY, USA) and R 4.0.2. with nine $R$ packages, including survival, survminer, tableone, broom, $P R O C, D M w R, R O C R$, CoxBoost, and randomForestSRC.

\section{Results}

\subsection{Baseline characteristics of the study population}

A total of 495 patients were included in the final analysis. To better explore the relationship between CCL17 and the prognosis of patients receiving coronary angiogram, the studied patients were divided into 3 groups according to CCL17 concentration: CCL17 low (CCL17 $\leq 185.960 \mathrm{pg} / \mathrm{mL}, \mathrm{N}=137$ ), CCL17 medium (185.960 pg/mL <CCL17 $\leq 268.173 \mathrm{pg} / \mathrm{mL}, \mathrm{N}=136)$, and CCL17 high (CCL17 > 268.173pg/mL, $\mathrm{N}=222$ ). The setting of cutoff concentrations was displayed in Supplementary Fig. 1.

As shown in Table 1, the patients in higher CCL17 groups showed significantly worse syndrome. With the elevation of CCL17, a higher Gensini score $(p=0.019)$ and more lesion vessels $(p<0.001)$ were recorded, indicating that a high CCL17 level was correlated with worse atherosclerotic lesions. Meanwhile, compared to CCL17 low groups, more patients in the other two groups were diagnosed with NSTEMI and STEMI, demonstrating a more serious cardiac injury. 
Table 1

Baseline characteristics of the study population

\begin{tabular}{|c|c|c|c|c|c|c|}
\hline & $\begin{array}{l}\text { All } \\
\text { patients } \\
(\mathrm{N}=495)\end{array}$ & $\begin{array}{l}\text { CCL17 low } \\
(N=137)\end{array}$ & $\begin{array}{l}\mathrm{CCL} 17 \\
\text { medium } \\
(\mathrm{N}=136)\end{array}$ & $\begin{array}{l}\text { CCL17 } \\
\text { high } \\
(N=222)\end{array}$ & $\begin{array}{l}\text { Statistic } \\
\text { Value }\end{array}$ & P-value \\
\hline Age, years * & $\begin{array}{l}61.38 \pm \\
10.37\end{array}$ & $\begin{array}{l}61.18 \pm \\
10.47\end{array}$ & $\begin{array}{l}61.82 \pm \\
10.44\end{array}$ & $\begin{array}{l}61.23 \pm \\
10.30\end{array}$ & 0.169 & 0.844 \\
\hline Sex, male $\S$ & 347 (70.1) & $93(67.8)$ & 95 (69.9) & $159(71.6)$ & 0.570 & 0.752 \\
\hline BMI, $\mathrm{kg} / \mathrm{m}^{2}$ * & $\begin{array}{l}25.79 \pm \\
3.48\end{array}$ & $\begin{array}{l}25.75 \pm \\
3.19\end{array}$ & $\begin{array}{l}25.45 \pm \\
3.68\end{array}$ & $\begin{array}{l}26.02 \pm \\
3.51\end{array}$ & 1.076 & 0.342 \\
\hline $\mathrm{SBP}, \mathrm{mmHg}$ * & $\begin{array}{l}128.5 \pm \\
17.1\end{array}$ & $\begin{array}{l}128.9 \pm \\
17.5\end{array}$ & $\begin{array}{l}129.7 \pm \\
18.4\end{array}$ & $\begin{array}{l}127.6 \pm \\
16.0\end{array}$ & 0.658 & 0.518 \\
\hline $\mathrm{DBP}, \mathrm{mmHg}$ * & $74.3 \pm 12.2$ & $75.6 \pm 12.5$ & $75.1 \pm 11.9$ & $73.1 \pm 12.1$ & 2.218 & 0.110 \\
\hline \multicolumn{7}{|l|}{ Medical History } \\
\hline Smoke $\S$ & $382(77.2)$ & $110(80.3)$ & $106(77.9)$ & $165(74.3)$ & 1.802 & 0.406 \\
\hline Drinking § & 154 (31.7) & $39(29.1)$ & $39(28.9)$ & $76(35.0)$ & 2.017 & 0.365 \\
\hline Hypertension § & 339 (68.5) & $87(63.5)$ & $97(71.3)$ & 159 (71.6) & 2.266 & 0.322 \\
\hline Dyslipidemia § & 338 (68.3) & $89(65.0)$ & $90(66.2)$ & 159 (71.6) & 2.118 & 0.347 \\
\hline Diabetes $\S$ & $181(36.6)$ & $48(35.0)$ & $44(32.4)$ & $89(40.1)$ & 2.368 & 0.306 \\
\hline \multicolumn{7}{|c|}{ Angiographic Data } \\
\hline Gensini score $†$ & $\begin{array}{l}32.0(15.0 \\
54.0)\end{array}$ & $\begin{array}{l}20.0(5.0 \\
43.0)\end{array}$ & $\begin{array}{l}34.0(18.0 \\
67.3)\end{array}$ & $\begin{array}{l}33.0(19.0 \\
53.0)\end{array}$ & 7.967 & $0.019^{b}$ \\
\hline $\begin{array}{l}\text { No. of lesion } \\
\text { vessels § }\end{array}$ & & & & & 27.956 & $\begin{array}{l}<.001^{a, b} \\
\text { a }\end{array}$ \\
\hline
\end{tabular}

Notes, * was the label for the continuous variables with normal distribution and were presented as mean $\pm S D$, analyzed via ANOVA with the $F$ value. The other continuous variables were labeled with $t$, presented as median (IQR), analyzed via the Kruskal-Wallis test, with the $\mathrm{H}$ value. Categorical variables were labeled with §, presented as $\mathrm{n}(\%)$, analyzed via Chi-squared test, with $\mathrm{x}^{2}$ value,

For comparison among groups, $a: p<0.05$ for equality between CCL 17 low vs. medium; $b: p<0.05$ for equality between CCL17 low vs. high; $c$ : $p<0.05$ for equality between CCL17 medium vs. high.

BMI: body mass index. SBP: systolic blood pressure. DBP: diastolic blood pressure. CHR: cardiovascular high-risk patients; SAP: stable angina pectoris. UA: Unstable angina. NSTEMI: non-ST segment elevation myocardial infarction. STEMI: ST-segment elevation myocardial infarction. WBC: white blood cell. PLT: platelet. TC: total cholesterol. LDLC: low-density lipoprotein cholesterol. HbA1c: glycosylated hemoglobin A1c. hsCRP: high sensitivity C-reactive protein. eGFR: estimated glomerular filtration rate. ALT: alanine aminotransferase. ACEl: angiotensin-converting enzyme inhibitor. ARB: angiotensin receptor blocker. PCl: percutaneous transluminal coronary intervention. CABG: coronary artery bypass grafting. 


\begin{tabular}{|c|c|c|c|c|c|c|}
\hline & $\begin{array}{l}\text { All } \\
\text { patients } \\
(\mathrm{N}=495)\end{array}$ & $\begin{array}{l}\text { CCL17 low } \\
(N=137)\end{array}$ & $\begin{array}{l}\text { CCL17 } \\
\text { medium } \\
(\mathrm{N}=136)\end{array}$ & $\begin{array}{l}\text { CCL17 } \\
\text { high } \\
(N=222)\end{array}$ & $\begin{array}{l}\text { Statistic } \\
\text { Value }\end{array}$ & P-value \\
\hline 0 & $53(10.7)$ & 25 (18.2) & 18 (13.3) & $10(4.5)$ & & \\
\hline 1 & 115 (23.2) & 38 (27.7) & $24(17.6)$ & $53 b(23.9)$ & & \\
\hline 2 & $120(24.2)$ & $24(17.5)$ & $28(20.6)$ & $68(30.6)$ & & \\
\hline 3 & 205 (41.4) & $50(36.5)$ & $64(47.1)$ & $91(40.9)$ & & \\
\hline Diagnosis & & & & & 21.293 & $0.006^{a, b}$ \\
\hline $\mathrm{CHR} \S$ & 57 (11.5) & $25(18.2)$ & $18(13.2)$ & $14(6.3)$ & & \\
\hline SAP § & $97(19.6)$ & $31(22.6)$ & $24(17.6)$ & $42(18.9)$ & & \\
\hline UA § & $211(42.6)$ & 57 (41.6) & $52(38.2)$ & $102(45.9)$ & & \\
\hline NSTEMI § & $50(10.1)$ & $11(8.0)$ & $19(14.0)$ & $20(9.0)$ & & \\
\hline STEMI § & 79 (16.0) & $13(9.5)$ & 23(16.9) & 43 (19.4) & & \\
\hline \multicolumn{7}{|c|}{ Laboratory Results } \\
\hline WBC, $10^{\wedge} 9 / \mathrm{L}$ * & $6.79 \pm 1.75$ & $6.61 \pm 1.72$ & $6.63 \pm 1.55$ & $6.99 \pm 1.87$ & 2.579 & 0.077 \\
\hline PLT, 10^9/L † & $\begin{array}{l}214(176, \\
255)\end{array}$ & $\begin{array}{l}196(155 \\
235)\end{array}$ & $\begin{array}{l}220(179 . \\
272)\end{array}$ & $\begin{array}{l}183(226 \\
258)\end{array}$ & 2.157 & 0.340 \\
\hline $\mathrm{TC}, \mathrm{mmol} / \mathrm{L} \dagger$ & $\begin{array}{l}4.04(3.52, \\
4.55)\end{array}$ & $\begin{array}{l}4.05(3.46, \\
4.55)\end{array}$ & $\begin{array}{l}3.86(3.47 \\
4.79)\end{array}$ & $\begin{array}{l}4.09(3.52, \\
4.54)\end{array}$ & 0.220 & 0.896 \\
\hline LDLc, mmol/L * & $2.27 \pm 0.92$ & $2.36 \pm 0.90$ & $2.29 \pm 0.92$ & $2.20 \pm 0.93$ & 1.361 & 0.257 \\
\hline $\mathrm{HbA1c}, \% †$ & $\begin{array}{l}6.15(5.70 \\
7.10)\end{array}$ & $\begin{array}{l}6.70(5.80 \\
8.00)\end{array}$ & $\begin{array}{l}6.00(5.65 \\
6.90)\end{array}$ & $\begin{array}{l}6.00(5.70 \\
7.00)\end{array}$ & 0.796 & 0.672 \\
\hline
\end{tabular}

Notes, * was the label for the continuous variables with normal distribution and were presented as mean $\pm S D$, analyzed via ANOVA with the $F$ value. The other continuous variables were labeled with $t$, presented as median (IQR), analyzed via the Kruskal-Wallis test, with the $\mathrm{H}$ value. Categorical variables were labeled with §, presented as $\mathrm{n}(\%)$, analyzed via Chi-squared test, with $\mathrm{x}^{2}$ value,

For comparison among groups, $a: p<0.05$ for equality between CCL17 low vs. medium; $b$ : $p<0.05$ for equality between CCL17 low vs. high; $c$ : $p<0.05$ for equality between CCL17 medium vs. high.

BMI: body mass index. SBP: systolic blood pressure. DBP: diastolic blood pressure. CHR: cardiovascular high-risk patients; SAP: stable angina pectoris. UA: Unstable angina. NSTEMI: non-ST segment elevation myocardial infarction. STEMI: ST-segment elevation myocardial infarction. WBC: white blood cell. PLT: platelet. TC: total cholesterol. LDLc: low-density lipoprotein cholesterol. HbA1c: glycosylated hemoglobin A1c. hsCRP: high sensitivity C-reactive protein. eGFR: estimated glomerular filtration rate. ALT: alanine aminotransferase. ACEl: angiotensin-converting enzyme inhibitor. ARB: angiotensin receptor blocker. PCl: percutaneous transluminal coronary intervention. CABG: coronary artery bypass grafting. 


\begin{tabular}{|c|c|c|c|c|c|c|}
\hline & $\begin{array}{l}\text { All } \\
\text { patients } \\
(\mathrm{N}=495)\end{array}$ & $\begin{array}{l}\text { CCL17 low } \\
(N=137)\end{array}$ & $\begin{array}{l}\text { CCL17 } \\
\text { medium } \\
(\mathrm{N}=136)\end{array}$ & $\begin{array}{l}\text { CCL17 } \\
\text { high } \\
(\mathrm{N}=222)\end{array}$ & $\begin{array}{l}\text { Statistic } \\
\text { Value }\end{array}$ & P-value \\
\hline hsCRP, mg/L † & $\begin{array}{l}1.47(0.68, \\
4.30)\end{array}$ & $\begin{array}{l}0.94(0.53 \\
2.98)\end{array}$ & $\begin{array}{l}1.72(0.73 \\
8.40)\end{array}$ & $\begin{array}{l}2.23(0.86 \\
4.92)\end{array}$ & 8.708 & $0.013^{b}$ \\
\hline $\begin{array}{l}\text { eGFR, } \\
\mathrm{mL} /\left(\min \star 1.73 \mathrm{~m}^{2}\right)\end{array}$ & $\begin{array}{l}87.5(70.4, \\
98.7)\end{array}$ & $\begin{array}{l}84.1(65.9, \\
97.3)\end{array}$ & $\begin{array}{l}94.6(79.3, \\
103)\end{array}$ & $\begin{array}{l}85.6(70.1 \\
98.7)\end{array}$ & 1.791 & 0.408 \\
\hline$A L T, U / L+$ & $\begin{array}{l}25.0(19.0 \\
41.0)\end{array}$ & $\begin{array}{l}26.0(22.5 \\
38.5)\end{array}$ & $\begin{array}{l}23.0(18.0, \\
43.0)\end{array}$ & $\begin{array}{l}26.0(19.0 \\
45.5)\end{array}$ & 1.043 & 0.594 \\
\hline \multicolumn{7}{|l|}{$\begin{array}{l}\text { Post-discharge } \\
\text { medication }\end{array}$} \\
\hline Statin $\S$ & 399 (91.1) & 103(83.1) & 107 (92.2) & $189(95.5)$ & 14.686 & $0.001^{a, b}$ \\
\hline ACEI/ARB § & $266(60.7)$ & $69(55.6)$ & $74(63.8)$ & $123(62.1)$ & 1.961 & 0.375 \\
\hline$\beta$-blocker $\S$ & $345(78.8)$ & $94(75.8)$ & $89(76.7)$ & $162(81.8)$ & 2.041 & 0.360 \\
\hline Aspirin $\S$ & $411(93.8)$ & $114(91.9)$ & $106(91.4)$ & $191(96.5)$ & 4.350 & 0.114 \\
\hline Warfarin $\S$ & $3(0.7)$ & $0(0)$ & $3(2.6)$ & $0(0)$ & 8.385 & 0.015 \\
\hline \multicolumn{7}{|l|}{ Operation history } \\
\hline $\mathrm{PCl} \S$ & $229(46.3)$ & $57(41.6)$ & $67(49.3)$ & $105(47.3)$ & 1.784 & 0.410 \\
\hline CABG $\S$ & $25(5.1)$ & $7(5.1)$ & $5(3.7)$ & $13(5.9)$ & 0.837 & 0.658 \\
\hline \multicolumn{7}{|c|}{$\begin{array}{l}\text { Notes, * was the label for the continuous variables with normal distribution and were presented as } \\
\text { mean } \pm \text { SD, analyzed via ANOVA with the } \mathrm{F} \text { value. The other continuous variables were labeled with }+ \text {, } \\
\text { presented as median (IQR), analyzed via the Kruskal-Wallis test, with the } \mathrm{H} \text { value. Categorical } \\
\text { variables were labeled with } \S \text {, presented as } \mathrm{n}(\%) \text {, analyzed via Chi-squared test, with } x^{2} \text { value, }\end{array}$} \\
\hline \multicolumn{7}{|c|}{$\begin{array}{l}\text { For comparison among groups, } a: p<0.05 \text { for equality between } C C L 17 \text { low vs. medium; } b \text { : } p<0.05 \text { for } \\
\text { equality between CCL } 17 \text { low vs. high; } c \text { : } p<0.05 \text { for equality between CCL } 17 \text { medium vs. high. }\end{array}$} \\
\hline \multicolumn{7}{|c|}{$\begin{array}{l}\text { BMI: body mass index. SBP: systolic blood pressure. DBP: diastolic blood pressure. CHR: } \\
\text { cardiovascular high-risk patients; SAP: stable angina pectoris. UA: Unstable angina. NSTEMI: non-ST } \\
\text { segment elevation myocardial infarction. STEMI: ST-segment elevation myocardial infarction. WBC: } \\
\text { white blood cell. PLT: platelet. TC: total cholesterol. LDLC: low-density lipoprotein cholesterol. HbA1c: } \\
\text { glycosylated hemoglobin A1c. hsCRP: high sensitivity C-reactive protein. eGFR: estimated glomerular } \\
\text { filtration rate. ALT: alanine aminotransferase. ACEl: angiotensin-converting enzyme inhibitor. ARB: } \\
\text { angiotensin receptor blocker. PCl: percutaneous transluminal coronary intervention. CABG: coronary } \\
\text { artery bypass grafting. }\end{array}$} \\
\hline
\end{tabular}

There was no statistical correlation between CCL17 and other traditional risk factors, such as age, blood pressure, serum cholesterol, PCI history, except hsCRP. CCL17 high group was featured with high hsCRP compared to CCL17 low group, pointing out the hyperinflammatory state of patients with high CCL17. 
In summary, a high CCL17 level demonstrated a hyperinflammatory state in patients at risk for CHD, which might aggravate the lesions and induce more severe symptoms. Meanwhile, CCL17 is also a risk factor independent from most other traditional cardiovascular risk factors.

\subsection{Clinical outcomes and Kaplan-Meier analysis based on CCL17 groups}

The prognostic data of the patients were collected through annual follow-up till December 2020 and the median follow-up time is 6.72 (IQR: $6.45,6.97$ ) years. The incidence of MACE was compared among groups of different CCL17 levels via chi-square test and summarized in Table 2. 116 (23.4\%) MACE were recorded, including 42 all-cause death (8.5\%), 15 recurrent myocardial infarction (Re-MI, 3.0\%), 21 angina (4.2\%), 5 heart failure (1.0\%), 7 ischemic cerebrovascular events (1.4\%), 4 hemorrhagic cerebrovascular events (0.8\%), 9 in-stent stenosis (1.8\%), 29 Re-PCI (5.9\%), 4 Re-CABG (0.8\%).

Table 2

Incidence of MACE according to CCL17 groups

\begin{tabular}{|c|c|c|c|c|c|}
\hline & $\begin{array}{l}\text { All } \\
\text { patients, } \\
\text { n (\%) }\end{array}$ & $\begin{array}{l}\text { CCL17 } \\
\text { low, } \\
\text { n (\%) }\end{array}$ & $\begin{array}{l}\text { CCL17 } \\
\text { medium, } \\
\mathrm{n}(\%)\end{array}$ & $\begin{array}{l}\text { CCL17 } \\
\text { high, } \\
\text { n (\%) }\end{array}$ & P-value \\
\hline MACE & $\begin{array}{l}116 \\
(23.4)\end{array}$ & $8(5.8)$ & $33(24.3)$ & 75 (33.8) & $\begin{array}{l}<0.001 \\
a, b\end{array}$ \\
\hline Death & $42(8.5)$ & $3(2.2)$ & $16(11.8)$ & $23(10.4)$ & $0.007^{a, b}$ \\
\hline Re-Ml & $15(3.0)$ & $2(1.5)$ & $2(1.5)$ & $11(5.0)$ & $0.079 b, c$ \\
\hline Angina & $21(4.2)$ & $0(0)$ & $3(2.2)$ & $18(8.1)$ & $\begin{array}{l}<.001^{\mathrm{a}, \mathrm{b}} \\
<\end{array}$ \\
\hline Heart Failure & $5(1.0)$ & $0(0)$ & $3(2.2)$ & $2(0.9)$ & 0.186 \\
\hline Ischemic Cerebrovascular event & $7(1.4)$ & $1(0.7)$ & $1(0.7)$ & $5(2.3)$ & 0.363 \\
\hline $\begin{array}{l}\text { Hemorrhagic Cerebrovascular } \\
\text { event }\end{array}$ & $4(0.8)$ & $1(0.7)$ & $1(0.7)$ & $2(0.9)$ & 0.979 \\
\hline In-stent stenosis & $9(1.8)$ & $0(0)$ & $1(0.7)$ & $8(3.6)$ & $0.025^{a, b}$ \\
\hline $\mathrm{Re}-\mathrm{PCl}$ & $29(5.9)$ & $2(1.5)$ & $7(5.1)$ & $20(9.0)$ & $0.012^{\mathrm{b}}$ \\
\hline Re-CABG & $4(0.8)$ & $1(0.7)$ & $1(0.7)$ & $2(0.9)$ & 0.979 \\
\hline
\end{tabular}

The incidence of all MACE, all-cause death, angina, in-stent stenosis, and Re-PCl increased significantly in patients in CCL17 medium and high groups compared with those in CCL17 low group (all chi-square $\mathrm{P}<$ 0.05). However, the Re-MI (chi-square $P=0.079$ ) and Re-CABG (chi-square $P=0.979$ ) were similar among the three groups, which may be caused by the relatively limited patient number. Other events, including 
chronic heart failure and cerebrovascular diseases, also had no significant differences among the three groups. Therefore, for patients at risk for CHD, an elevated CCL17 may indicate a poorer prognosis and higher risk of acute cardiac syndromes in the future. It is noteworthy that there is no significant difference in MACE incidences between CCL17 medium and high groups (Table 2).

Kaplan-Meier curves for the incidence of all MACE and all-cause death according to different CCL17 levels were shown in Fig. 2. Significant differences were observed in Kaplan-Meier curves for both incidence of all MACE (Fig. 2A, Log-rank $p<0.001$ ) and deaths (Fig. 2B, Log-rank $p=0.046$ ) between the CCL17 low group and other two groups.

The predictive value of CCL17 level on MACE and all-cause death was evaluated by univariate Cox proportional hazard analysis. The results showed that compared to the CCL17 low group, the risk of MACE increases 4.51 (95\% Cl 2.08-9.77) and 6.61 (95\% Cl 3.20-13.70) times respectively in CCL17 medium and high groups (Table 3 ).

Table 3

Incidence of MACE according to the optimal cutoff point of CCL17 level

\begin{tabular}{|c|c|c|c|c|c|}
\hline \multirow[t]{2}{*}{ Models } & \multirow[t]{2}{*}{ CCL17 low } & \multicolumn{2}{|l|}{ CCL17 medium } & \multicolumn{2}{|l|}{ CCL17 high } \\
\hline & & $\mathrm{HR}(95 \% \mathrm{Cl})$ & P-value & $\mathrm{HR}(95 \% \mathrm{Cl})$ & P-value \\
\hline Crude Model & 1 (Referent) & $4.51(2.08-9.77)$ & 0.00013 & $6.61(3.20-13.70)$ & $P<0.00001$ \\
\hline Model 1 & 1 (Referent) & $4.42(2.04-9.57)$ & 0.00017 & $6.65(3.2-13.77)$ & $P<0.00001$ \\
\hline Model 2 & 1 (Referent) & $4.22(1.94-9.18)$ & 0.00028 & $6.24(3.01-12.98)$ & $P<0.00001$ \\
\hline Model 3 & 1 (Referent) & $4.53(2.08-9.88)$ & 0.00015 & $6.38(3.07-13.28)$ & $P<0.00001$ \\
\hline \multicolumn{6}{|c|}{$\begin{array}{l}\text { The prediction ability of CCL17 onto the incidence of MACE was analyzed via univariate or } \\
\text { multivariate Cox proportional hazard analysis. }\end{array}$} \\
\hline \multicolumn{6}{|c|}{ Crude model: univariate Cox proportional hazard analysis. } \\
\hline \multicolumn{6}{|c|}{ Model 1: adjusted for Age + Sex + BMI. } \\
\hline \multicolumn{6}{|c|}{ Model 2: adjusted for Age + Sex + BMI + Diabetes + Hypertension + Number of lesion vessels. } \\
\hline \multicolumn{6}{|c|}{$\begin{array}{l}\text { Model 3: adjusted for Age + Sex + BMI + Diabetes + Hypertension + Number of lesion vessels + PLT + } \\
\text { hsCRP + eGFR + ALT. }\end{array}$} \\
\hline \multicolumn{6}{|c|}{$\begin{array}{l}\text { HR: hazard ratio. Cl: confidence interval. BMI: body mass index. PLT: platelet count. hsCRP: high } \\
\text { sensitivity C-reactive protein. eGFR: estimated glomerular filtration rate. ALT: alanine } \\
\text { aminotransferase. }\end{array}$} \\
\hline
\end{tabular}

Since cardiovascular diseases are complex and multiple risk factors have been well summarized, we also analyzed the conventional risk factors in our cohort (Supplementary Table 1). Some conventional risk factors including age, sex, smoking, dyslipidemia history, and hypertension history did not demonstrate much correlation with the occurrence of MACE, which may be due to a good drug intervention and medication compliance. The number of lesion vessels, disease history of diabetes, and abnormal 
laboratory test indexes such as platelet count, $\mathrm{HbA1c}$, hsCRP concentration showed a positive correlation with MACE, while eGFR showed a negative correlation with MACE (Supplementary Table 1).

\subsection{Cox proportional hazard analysis to evaluate the prognostic implication of CCL17}

In multivariate Cox proportional hazard analysis, three models (Model 1, 2,3) adjusted for different covariates were constructed to evaluate the prediction ability of CCL17 for all MACE. The variables included in the 3 models were selected based on clinical experience and statistical significance from results in Supplementary Table 1. In all 3 models, a higher CCL17 level remained as an independent risk predictor of all MACE (Table 4, all $p<0.001$ ). And model 3 , the full-adjusted model, described that in CCL17 medium and high groups, the risk of MACE increased to $4.53(95 \% \mathrm{Cl} 2.08-9.88)$ and $6.38(95 \% \mathrm{Cl}$ 3.07-13.28) times respectively, compared to CCL17 low group (Table 3).

Table 4

AUC and $\mathrm{C}$ index value of different models

\begin{tabular}{|lllllll|}
\hline & AUC & \multicolumn{5}{c|}{ C index } \\
\hline & $\begin{array}{l}\text { Without } \\
\text { CCL17 }\end{array}$ & With CCL17 & P-value & Without CCL17 & With CCL17 & P-value \\
\hline Model 1 & & & & & & \\
\hline Cox & $0.572 \pm 0.026$ & $0.655 \pm 0.022$ & $<0.001$ & $0.569 \pm 0.016$ & $0.654 \pm 0.016$ & $<0.001$ \\
\hline Coxboost & $0.557 \pm 0.017$ & $0.605 \pm 0.011$ & $<0.001$ & $0.537 \pm 0.011$ & $0.569 \pm 0.007$ & $<0.001$ \\
\hline RF2000 & $0.702 \pm 0.022$ & $0.734 \pm 0.021$ & $<0.001$ & $0.652 \pm 0.015$ & $0.672 \pm 0.014$ & $<0.001$ \\
\hline Model 2 & & & & & & \\
\hline Cox & $0.631 \pm 0.025$ & $0.667 \pm 0.025$ & $<0.001$ & $0.618 \pm 0.018$ & $0.685 \pm 0.018$ & $<0.001$ \\
\hline Coxboost & $0.582 \pm 0.015$ & $0.626 \pm 0.009$ & $<0.001$ & $0.552 \pm 0.01$ & $0.58 \pm 0.007$ & $<0.001$ \\
\hline RF2000 & $0.773 \pm 0.021$ & $0.819 \pm 0.02$ & $<0.001$ & $0.697 \pm 0.014$ & $0.724 \pm 0.013$ & $<0.001$ \\
\hline Model 3 & & & & & & \\
\hline Cox & $0.627 \pm 0.024$ & $0.678 \pm 0.023$ & $<0.001$ & $0.631 \pm 0.018$ & $0.694 \pm 0.019$ & $<0.001$ \\
\hline Coxboost & $0.591 \pm 0.014$ & $0.621 \pm 0.011$ & $<0.001$ & $0.559 \pm 0.01$ & $0.577 \pm 0.008$ & $<0.001$ \\
\hline RF2000 & $0.803 \pm 0.021$ & $0.836 \pm 0.021$ & $<0.001$ & $0.718 \pm 0.013$ & $0.737 \pm 0.013$ & $<0.001$ \\
\hline
\end{tabular}

\subsection{Incremental Effect Of Ccl17 On Mace Prediction}

To better evaluate the effectiveness and accuracy of different models, their statistical significance and clinical importance were assessed by area under curve (AUC) and C index (Table 4, Fig. 3). Considering the existence of data imbalanced problem (patients with MACE vs patients without MACE: 116 vs 379), 
which limited the performance of models, CoxBoost and RF with 2000 trees(RF2000) were applied to optimize models as mentioned in the method part. RF2000 was shown to effectively enhance the performance of the models (Fig. 3 , blue lines.)

For baseline risk models (model 1, 2, 3) with RF2000 optimization, the number of covariates has increased from 3 to a maximum of 10 , which increased the AUC (from $0.702 \pm 0.026$ to $0.803 \pm 0.021$ ).

Notably, the addition of CCL17 significantly increased AUC value in all models (Fig. 3, models without CCL17 vs models with CCL17: full line vs dotted line, $p<0.001$ ), which enhances the prediction ability of all three kinds of baseline risk models. Meanwhile, the addition of CCL17 increases the C-index from $0.718 \pm 0.013$ to $0.737 \pm 0.013$ (Table 4), suggesting a significantly increased model accuracy.

The statistical calculation results exhibited a strong prediction potential to MACE of CCL17. The model with the best prediction ability to MACE was also constructed with RF2000 optimization, 10 conventional risk factors, and CCL17 (AUC $0.836 \pm 0.021$ )

\section{Discussion}

CCL17 functions as an important inflammatory marker modulating inflammation by regulating the activity and recruitment of T cells[11-13,17]. Reports were discussing the relationship between CCL17 and various diseases. But to our knowledge, there are only a limited number of reports talking about CCL17 and CHD, and there was no previous report concerning the association between CCL17 and the prognosis of patients at CHD risk.

In this study, by collecting clinical information and completing long-term follow-up, the characteristics of 495 patients who underwent coronary angiography were summarized. Our cohort study indicated that elevated CCL17 level is closely related to the severity of coronary atherosclerosis. Meanwhile, serum CCL17 level was an independent predictor for the incidence of MACE after adjusting for confounding risk factors.

Several models were constructed to predict the prognosis of these patients. Nevertheless, the performance of the models was limited by the unbalanced patient number between patients with MACE and patients without MACE. To solve the problem, SMOTE was applied to optimize MACE prediction models, and RF2000 was proven to remarkably strengthen the efficiency of models. Then the addition of CCL17 was proven to significantly increase the efficiency of all models, confirming the prediction potency of CCL17 on the occurrence of MACE. Finally, the model with conventional CHD risk factors, CCL17, and RF2000 optimization was constructed, showing an AUC of $0.836 \pm 0.021$.

Notably, CCL17 displayed diverse effects on different sub-categories of MACE. For the events, including death, angina, in-stent stenosis, and re-PCl, statistical difference was observed between different CCL17 groups. But the effect of CCL17 on cerebrovascular events was inconspicuous. 
Moreover, although CCL17 medium and CCL17 high group showed an increased risk of MACE, the difference between these two groups was not significant. This suggested that CCL17 could increase the risk of death when the concentration is higher than a certain threshold, yet it is not linearly associated with the incidence rate of death.

There were multiple potential mechanisms through which CCL17 influencing the progression and prognosis of patients. Most importantly, as reported in the mouse model, CCL17 secreted from DCs could restrain Tregs in lymphatic tissue, which increased $T$ cells mediated inflammatory response and facilitated the formation of atherosclerotic lesions[11]. Besides, CCL17 was also reported to be expressed on endothelial cells, which might help to recruit and assemble circulating T cells to worsen lesions [24]. CCL17 could also result in a hyperinflammatory state of the organism and further lead to hypercoagulability and proliferation of smooth muscle cells, which was closely interrelated to in-stent stenosis[25,26]. However, the exact long-term impact of CCL17 on CHD still required more proof from basic researches

However, limitations also existed in this research. The size of this cohort limits the strength of the conclusion, and for several MACE sub-categories, such as myocardial infarction and Re-CABG, the insignificant result may be induced by the limited patient number. Further multi-center studies with an extended sample size was required to further confirm the role CCL17 played in patients at CHD risk. Most mechanism studies were also restricted to animal and cell experiments. How CCL17 affected MACE incidence and why certain sub-categories of MACE were more susceptible to elevated CCL17 levels is worthy of further exploration.

This research uncovers a novel marker that functions as a strong predictor for MACE and certain subcategories of MACE in patients who underwent coronary angiography. The existing basic mechanism research regarding CCL17 also implies its potency to exacerbate atherosclerotic lesions in patients directly. This research suggests a more aggressive drug treatment strategy could be applied to patients with high CCL17 to prevent the happening of future MACE. There are no available drug targeting CCL17 now, but an unspecific anti-inflammatory therapy may be applied to these patients, whose efficiency requires further clinical experiments.

\section{Conclusion}

This research identified a novel inflammation related marker CCL17 as a risk predictor of major adverse cardiovascurlaer events in patients underwent coronary angiogram. Besides, compared to conventional cardiovascular risk factors, the addition of CCL17 can dramatically ehance the prediction ability of different models for MACE, suggesting the prediction ability of CCL17. The exact mechanism exploration is required to further understand how CCL17 impacts patients.

\section{Declarations}


Acknowledgements

We acknowledge Dr. Yicong Ye and Dr. Xinglin Yang for helping collect clinical information and patients' sample.

\section{Authors' contributions}

SQF, YFW, YYW, RT, HW and HHL completed clinical information collection, patietnss sample measurement and follow-up. SQF, LW, YFW and YXC completed the statistical analysis. SQF and SYZ completed the study design. SQF, LW, YFW and YXC compelted manuscript writing. The study was completed under the guide of SYZ. All authors read and approved the final manuscript.

\section{Funding:}

This work was supported by CAMS Innovation Fund for Medical Sciences (CIFMS) [No. 2016-I2M-3-011, 2016-12M-1-002].

\section{Availability of data and materials}

The datasets used and/or analyzed during the current study are available from the corresponding author on reasonable request

\section{Ethics statement:}

This study was approved by the ethics committee of Peking Union Medical College Hospital. Signed informed consent was obtained from all study participants.

\section{Competing interests:}

The authors declare that they have no competing interests.

\section{Consent for publication}

Not applicable.

\section{References}

1. Virani SS, et al. Heart Disease and Stroke Statistics-2020 Update: A Report From the American Heart Association. Circulation. 2020;141(9):e139-596.

2. Mendis S, Davis S, Norrving B. Organizational update: the world health organization global status report on noncommunicable diseases 2014; one more landmark step in the combat against stroke and vascular disease. Stroke. 2015;46(5):e121-2.

3. Thomas H, et al. Global Atlas of Cardiovascular Disease 2000-2016: The Path to Prevention and Control. Glob Heart. 2018;13(3):143-63. 
4. Badimon L, Padró T, Vilahur G. Atherosclerosis, platelets and thrombosis in acute ischaemic heart disease. Eur Heart J Acute Cardiovasc Care. 2012;1(1):60-74.

5. Ibanez B, Vilahur G, Badimon JJ. Plaque progression and regression in atherothrombosis. J Thromb Haemost. 2007;5(Suppl 1):292-9.

6. Zernecke A, Weber C. Inflammatory mediators in atherosclerotic vascular disease. Basic Res Cardiol. 2005;100(2):93-101.

7. Malakar AK, et al. A review on coronary artery disease, its risk factors, and therapeutics. J Cell Physiol. 2019;234(10):16812-23.

8. Witztum JL, Lichtman AH. The influence of innate and adaptive immune responses on atherosclerosis. Annu Rev Pathol. 2014;9:73-102.

9. Tabas I, Lichtman AH. Monocyte-Macrophages and T Cells in Atherosclerosis. Immunity. 2017;47(4):621-34.

10. Alferink J, et al. Compartmentalized production of CCL17 in vivo: strong inducibility in peripheral dendritic cells contrasts selective absence from the spleen. J Exp Med. 2003;197(5):585-99.

11. Weber $\mathrm{C}$, et al. CCL17-expressing dendritic cells drive atherosclerosis by restraining regulatory $\mathrm{T}$ cell homeostasis in mice. J Clin Invest. 2011;121(7):2898-910.

12. Scheu S, et al., The C-C Chemokines CCL 17 and CCL22 and Their Receptor CCR4 in CNS Autoimmunity. Int J Mol Sci, 2017. 18(11).

13. Griffith JW, Sokol CL, Luster AD. Chemokines and chemokine receptors: positioning cells for host defense and immunity. Annu Rev Immunol. 2014;32:659-702.

14. Greaves DR, et al., Linked chromosome $16 q 13$ chemokines, macrophage-derived chemokine, fractalkine, and thymus- and activation-regulated chemokine, are expressed in human atherosclerotic lesions. Arterioscler Thromb Vasc Biol, 2001. 21(6): p. 923-9.

15. Feng $S$, et al. Plasma levels of monokine induced by interferon-gamma/chemokine (C-X-X motif) ligand 9, thymus and activation-regulated chemokine/chemokine (C-C motif) ligand 17 in children with Kawasaki disease. BMC Pediatr. 2015;15:109.

16. Stutte S, et al. Requirement of CCL17 for CCR7- and CXCR4-dependent migration of cutaneous dendritic cells. Proc Natl Acad Sci U S A. 2010;107(19):8736-41.

17. Heiseke AF, et al. CCL17 promotes intestinal inflammation in mice and counteracts regulatory T cellmediated protection from colitis. Gastroenterology. 2012;142(2):335-45.

18. Ye Y, et al. Association between serum chemokine CC-motif ligand 17 and coronary artery disease. Int J Cardiol. 2014;176(2):541-2.

19. Ye Y, et al. Serum chemokine CCL17/thymus activation and regulated chemokine is correlated with coronary artery diseases. Atherosclerosis. 2015;238(2):365-9.

20. Thygesen K, et al. Fourth Universal Definition of Myocardial Infarction (2018). J Am Coll Cardiol. 2018;72(18):2231-64. 
21. Blagus R, Lusa L. SMOTE for high-dimensional class-imbalanced data. BMC Bioinformatics. 2013;14:106.

22. Binder H. CoxBoost: Cox models by likelihood based boosting for a single survival endpoint or competing risks. 2013.

23. Shimokawa T, Tsuji M, Regularized Random Forest Method for Survival Analysis. leice Technical Report, 2010. 110: p. 71-77.

24. D'Ambrosio D, et al. Quantitative differences in chemokine receptor engagement generate diversity in integrin-dependent lymphocyte adhesion. J Immunol. 2002;169(5):2303-12.

25. Yang $D$, et al. Proliferation of vascular smooth muscle cells under inflammation is regulated by NFKB p65/microRNA-17/RB pathway activation. Int J Mol Med. 2018;41(1):43-50.

26. Posma JJ, et al. Roles of Coagulation Proteases and PARs (Protease-Activated Receptors) in Mouse Models of Inflammatory Diseases. Arterioscler Thromb Vasc Biol. 2019;39(1):13-24.

\section{Supplementary Materials}

Supplemental table 1 is not available with this version.

\section{Figures}


Patients undergoing coronary arteriography in a single academic center during 2012/01-2013/12 were recruited, with clinical information collected after signing informed consents

$(\mathrm{N}=1022)$

Reject participating long-term research program: 235

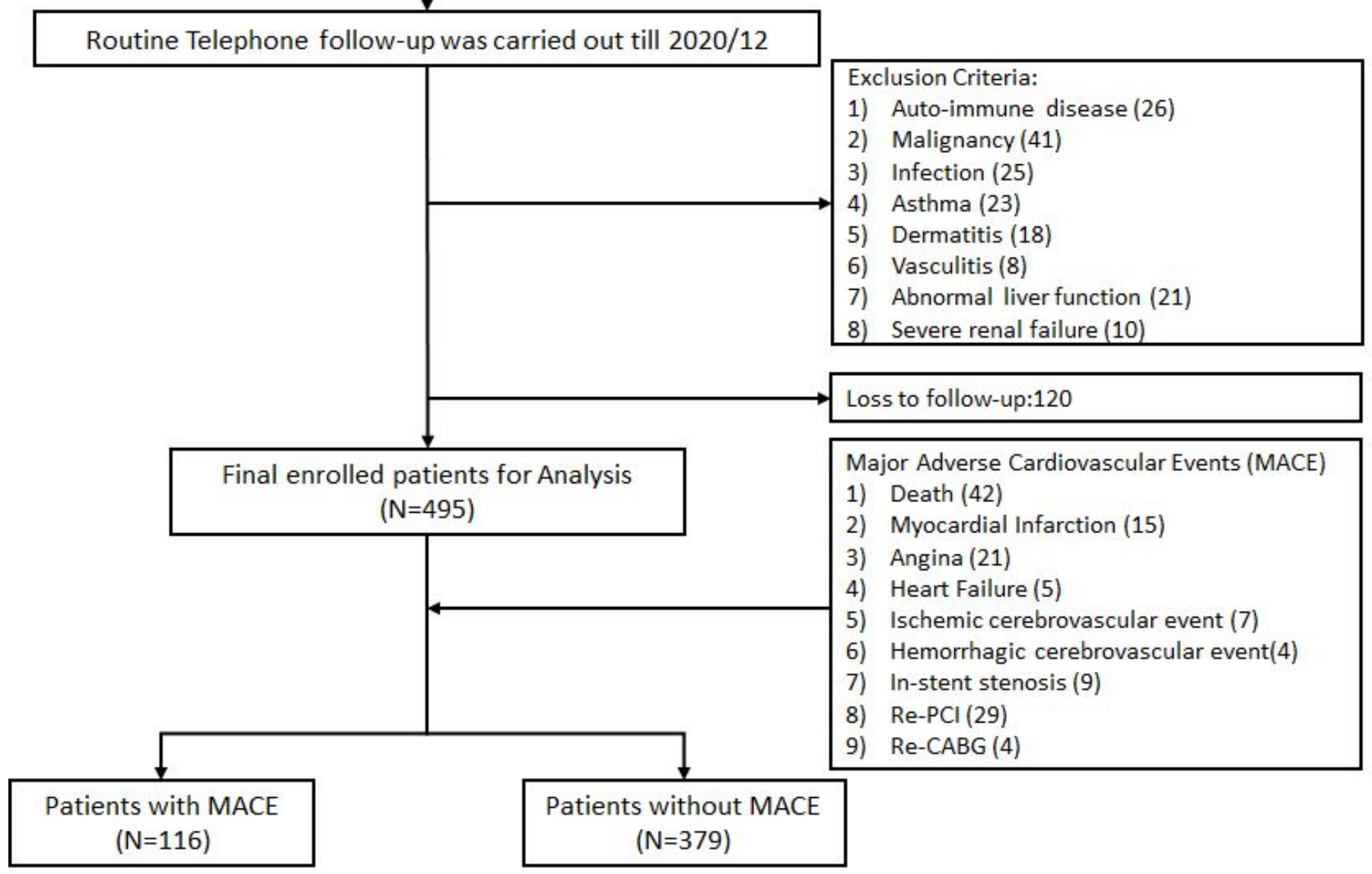

\section{Figure 1}

Flow chart of the study population enrollment 
A
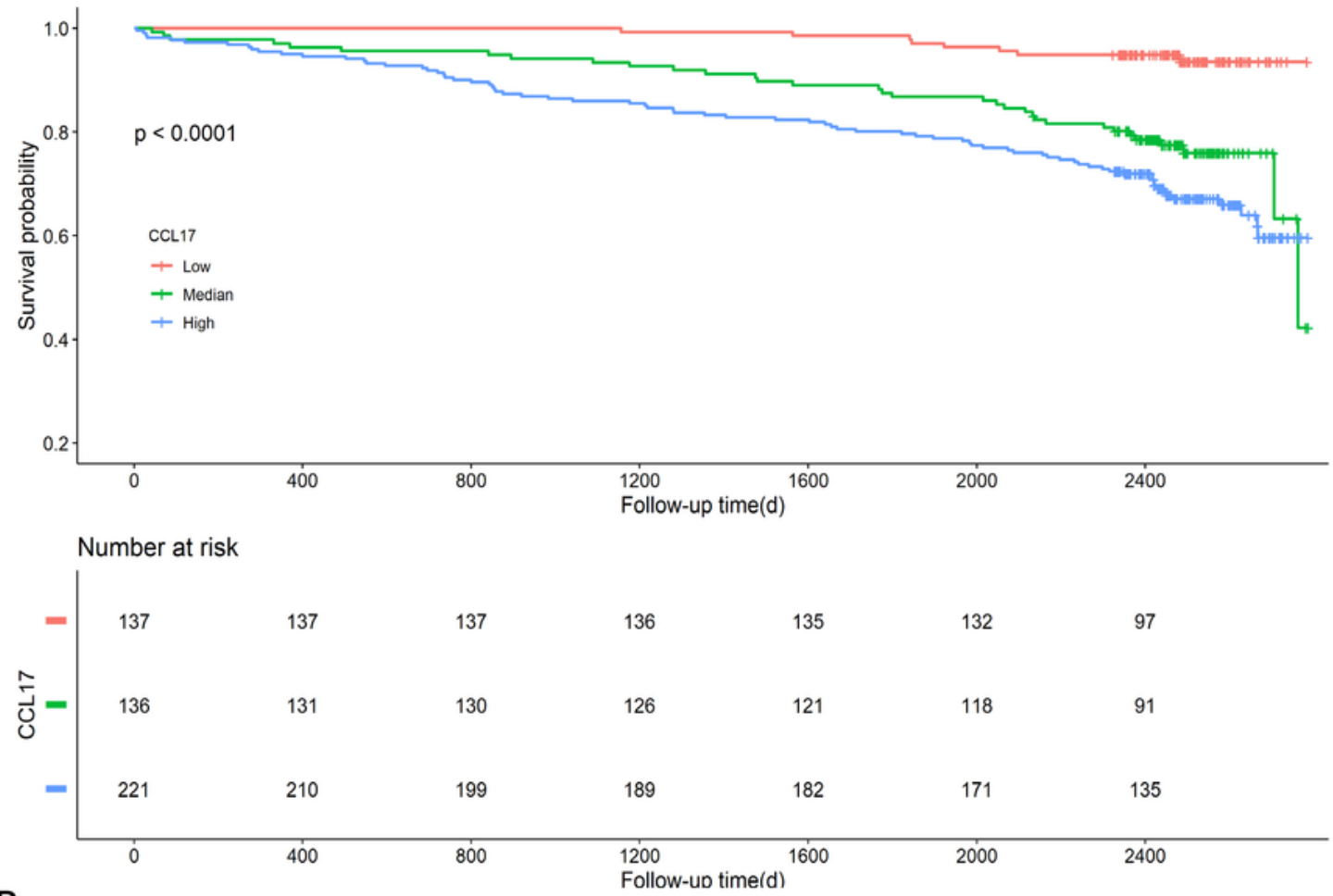

B

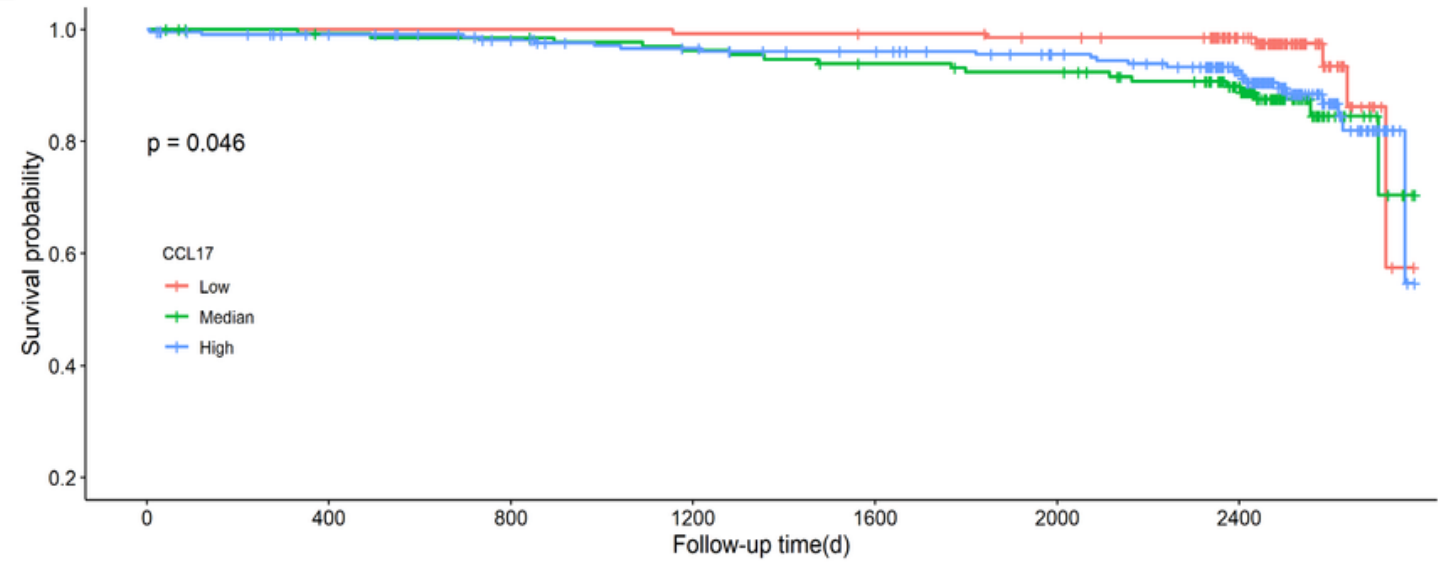

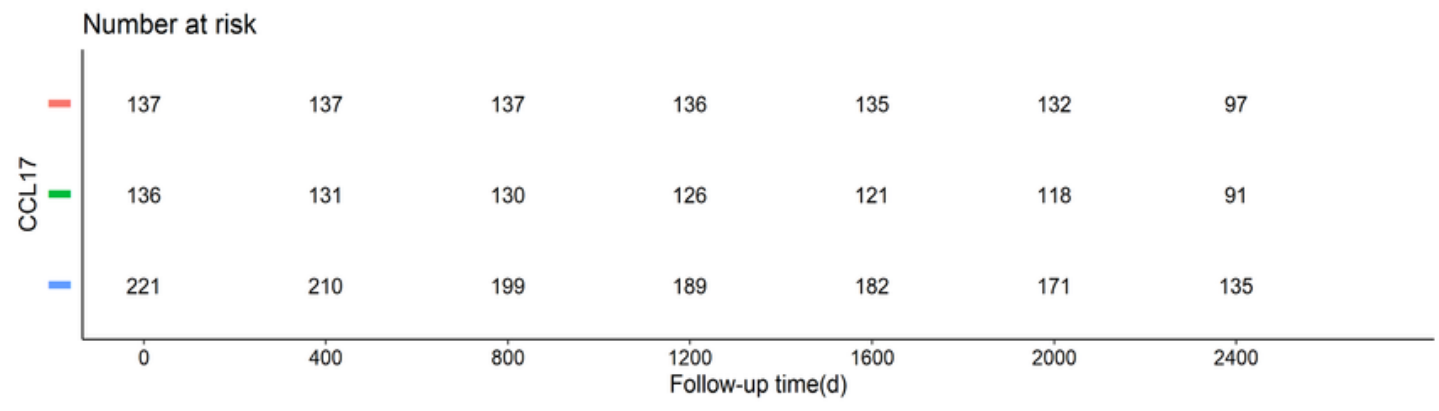

Figure 2

Kaplan-Meier curves for MACE and death according to CCL17 level A. Kaplan-Meier curves for the happen of all MACE. B. Kaplan-Meier curves for all-cause death. 

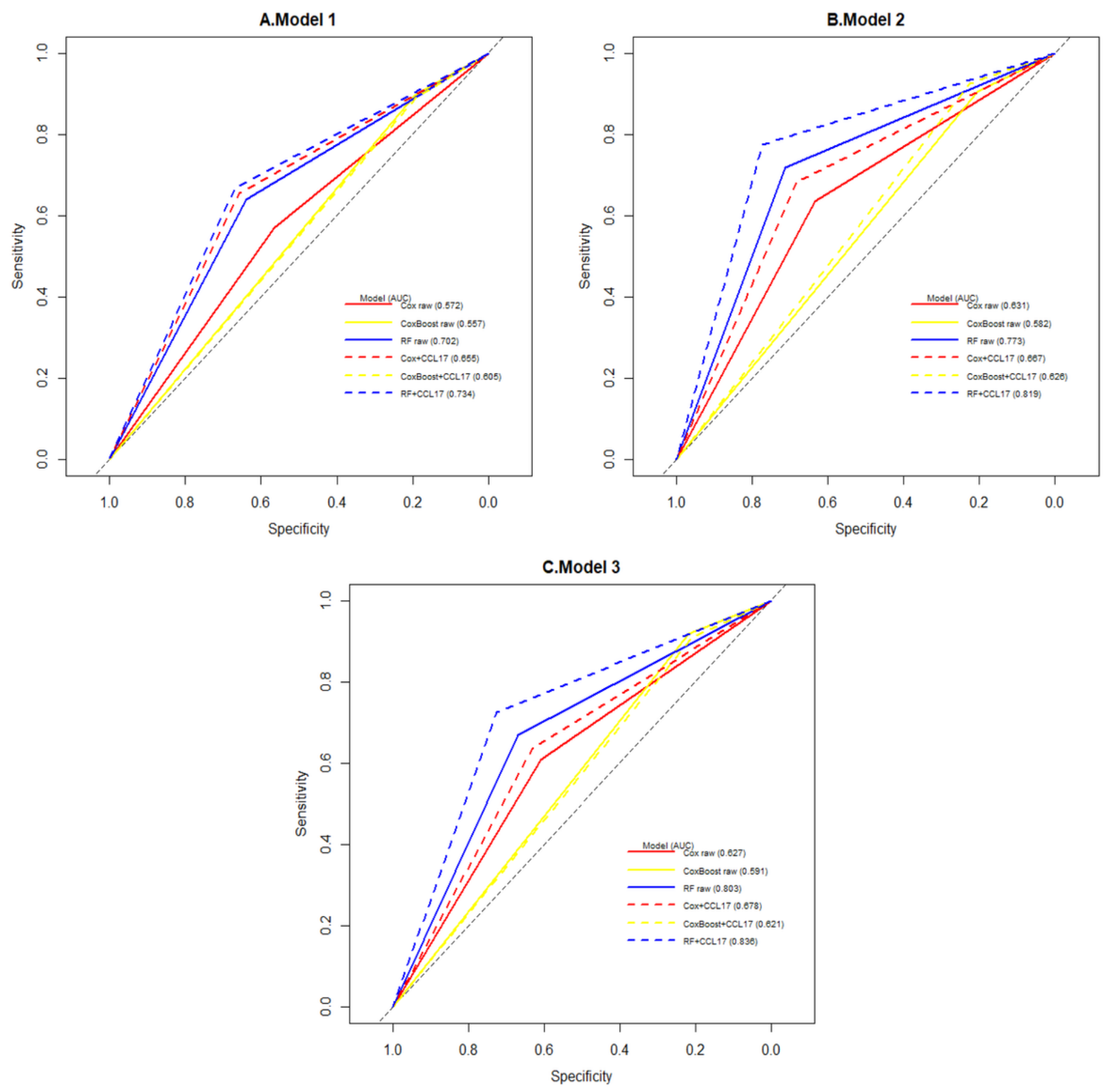

Figure 3

ROC of different models with or without CCL17 ROC curve of Cox is shown in the red line, CoxBoost in the yellow line, and RF2000 in the blue line. Models without CCL17 were drawn in the full line while models with CCL17 drawn in the dotted line. In different models, the number of covariates was increased in sequence model 1 (A), model 2 (B), and model 3 (C). The pattern is similar. The raw model calculated via Cox proportional hazard analysis was shown in a red full line, with Cox Boost optimization (yellow full line) decreasing AUC while RF2000 optimization (blue full line) increasing AUC. For each model and each optimization, the addition of CCL17 (dotted lines) can further increase AUC. 


\section{Supplementary Files}

This is a list of supplementary files associated with this preprint. Click to download.

- SuppFigure1.jpg 